\title{
Desempenho de cordeiros desmamados aos 67 dias alimentados com silagem de milho e feno de aveia
}

\author{
Performance of lambs weaned at 67 days of \\ age and fed corn silage and oat hay
}

\author{
Edson Luis de Azambuja Ribeiro ${ }^{1 *}$; Marco Antonio da Rocha ${ }^{1}$; \\ Ivone Yurika Mizubuti ${ }^{1}$; Leandro das Dores Ferreira da Silva ${ }^{1}$; \\ Sérgio Rodrigo Fischer²; Adriana Pereira da Silva ${ }^{2}$
}

\begin{abstract}
Resumo
Este trabalho teve como objetivo avaliar o desempenho de cordeiros desmamados aos 67 dias e alimentados com diferentes fontes de volumosos em confinamento. Foram utilizados 30 animais oriundos de cruzamentos entre ovelhas Corriedale e carneiros Hampshire Down, Ile de France e Suffolk. Logo após o desmame os animais foram confinados, sendo distribuídos para receberem um dos tratamentos: T1 - Silagem de milho mais concentrado; T2 - Silagem de milho e feno de aveia (50\% do volumoso cada) mais concentrado; e T3 - Feno de aveia mais concentrado. A proporção volumoso:concentrado utilizada foi de 60:40, base seca. Os pesos médios dos animais no início do experimento foram de 11,51; 12,46 e 12,33 kg (P>0,05) e os pesos finais, após nove semanas de confinamento, de 17,53; 19,34 e 19,35 kg (P>0,05), respectivamente, para T1, T2 e T3. Os animais apresentaram ganho de peso similar $(\mathrm{P}>0,05)$ entre os tratamentos. Não foram observadas diferenças para os pesos e ganhos de peso entre grupos raciais, porém, os machos apresentaram maiores ganhos de peso do que as fêmeas $(0,120 \times 0,091 \mathrm{~kg} / \mathrm{dia})$. Não houve diferença para a conversão alimentar entre os tratamentos, porém animais do T2 consumiram maior quantidade de matéria seca. Os resultados obtidos neste estudo sugerem que pode ser utilizado tanto a silagem de milho como o feno de aveia na alimentação de cordeiros desmamados, sem prejuízos na produção.
\end{abstract}

Palavras-chave: Confinamento, ganho de peso, grupo genético, ovinos, volumoso.

\begin{abstract}
This work had as objectives to evaluate the performance of lambs weaned at 67 days of age and fed with different roughage sources in confinement. Thirty animals out of Corriedale ewes mated to Hampshire Down, Ile de France and Suffolk rams, were used. After weaning the animals were fedlot and distributed in one of the following treatments: T1 - Corn silage + concentrate; T2 - Corn silage and oat hay $(50 \%$ each) + concentrate; and T3 - Oat hay + concentrate. It was used a 60:40 roughage:concentrate ratio, dry matter basis. At the beginning of the experiment the animals weighed $11.51,12.46$ and $12.33 \mathrm{~kg}(\mathrm{P}>0.05)$, and after nine weeks of feedlot the weights were 17.53, 19.34 and $19.35 \mathrm{~kg}(\mathrm{P}>0.05)$, respectively, for $\mathrm{T} 1$, $\mathrm{T} 2 \mathrm{e}$ T3. Average daily weight gain was similar $(\mathrm{P}>0.05)$ among treatments. It was not observed differences $(\mathrm{P}>0.05)$ in weights and weight gains among the genetic groups. However, male lambs presented greater average daily weight gain than female lambs $(0.120 \mathrm{x} 0.091 \mathrm{~kg})$. There were no differences in feed conversion among the treatments, however, animals from the $\mathrm{T} 2 \mathrm{had}$ greater dry matter intake. It can be concluded that either corn silage or oat hay can be used for feeding weaned lambs.
\end{abstract}

Key words: Feedlot, genetic group, roughage, sheep, weight gain.

\footnotetext{
${ }^{1}$ Professores do Departamento de Zootecnia da Universidade Estadual de Londrina. CEP 86051-990, Londrina, PR.

${ }^{2}$ Engenheiro(a) Agrônomo(a).

* Autor para correspondência.
} 


\section{Introdução}

A produção de carne ovina no Brasil apresenta grande potencial de crescimento, pois há um grande mercado consumidor não atendido nos centros urbanos. Em algumas regiões brasileiras, como no norte do Paraná, onde as condições de solo e climáticas favorecem a produção de pastagens com alto potencial produtivo, há a possibilidade de um aumento significativo na atividade. Porém, estas boas características climáticas, também, são favoráveis à manutenção de elevada população de helmintos nas pastagens. Este fato pode prejudicar a criação de animais jovens e de ovelhas em final de gestação e em lactação, pois estas duas categorias são as mais susceptíveis à verminose (AMARANTE, 1990). Siqueira (1996) cita que a recria e terminação de cordeiros em confinamento é uma opção para a intensificação da exploração de ovinos. $\mathrm{O}$ autor comenta que o confinamento, também, evita perdas econômicas decorrentes de baixos ganhos de peso e da mortalidade causada pela verminose.

Sistemas de terminação e recria de cordeiros tem sido estudado por alguns pesquisadores. Siqueira, Amarante e Fernandes (1993) observaram que cordeiros desmamados aos 60 dias de idade e confinados após o desmame, apresentaram peso $42 \%$ maior aos 150 dias de idade do que os cordeiros que permaneceram em pastagem após o desmame. A diferença nos ganhos de peso, segundo os autores, ocorreu pela menor infecção por nematódeos gastrintestinais no grupo confinado. $\mathrm{O}$ grupo que permaneceu em pastagem apresentou 16,23\% de mortalidade, enquanto no grupo confinado a mortalidade foi $0 \%$. Resultados concordantes com estes foram apresentados por Macedo, Siqueira e Martins (1998a) que observaram ganhos médios diários de $0,144 \mathrm{~kg}$ para cordeiros confinados e de 0,106 para cordeiros terminados em pastagem. Os autores concluíram que os cordeiros apresentam melhor terminação em confinamento, porém salientam que a adoção deste sistema depende de uma criteriosa análise dos custos envolvidos. Macedo, Siqueira e Martins (1998b) observaram melhor retorno econômico em cordeiros terminados em confinamento do que os terminados em pastagem. Siqueira, Amarante e Fernandes (1993) citam que a utilização de pastagens descontaminadas pode ser uma boa alternativa para a recria de cordeiros.

Para que o confinamento tenha sucesso é importante que, além de ser economicamente viável, sejam utilizados animais com potencial para ganho de peso e que a alimentação tenha qualidade suficiente para atender os requerimentos dos animais. $\mathrm{O}$ uso de forragens conservadas, tais como silagem de milho (Zea mays L.) e feno de aveia (Avena sativa L.), quando cultivadas apropriadamente e em estádios vegetativos recomendados, apresentam boas características químico-bromatológicas (SÁ, 1995; LUPATINI; NUNES, 1999).

Codagnone et al. (1988) compararam o uso da silagem de milho com feno de aveia em cinco proporções diferentes na alimentação de vacas leiteiras e não observaram diferenças no teor de gordura e na produção de leite. As proporções utilizadas, em percentagem de matéria seca dos volumosos, foram 100 e $0 \%$; 75 e $25 \%$; 50 e $50 \%$; 25 e $75 \%$ e 0 e $100 \%$, respectivamente, para silagem de milho e feno de aveia. De maneira semelhante, Ribeiro (2000) não observou diferenças no consumo de matéria seca, na produção e na composição do leite de cabras alimentadas com dietas onde a fonte volumosa foi silagem de milho ou feno de aveia. Por outro lado, na alimentação de ovinos, alguns cuidados devem ser tomados quanto à aceitação dos alimentos pelos animais. Milligan (1985) cita que as silagens são pouco apetecíveis para os ovinos, resultando em baixo consumo destes alimentos e conseqüentemente, níveis de produção aquém do esperado.

O potencial para ganho de peso está, também, relacionado com o genótipo dos animais. Em boas condições alimentares, animais provenientes de raças especializadas para corte ou de cruzamentos com estas raças, normalmente apresentam maiores ganhos de peso e pesos ao abate (CARTER; KIRTON, 1975; PILAR et al., 1994; MACEDO; 
SIQUEIRA; MARTINS, 1998a). Por outro lado, Pilar et al. (1994) não observaram diferenças no ganho de peso de ovinos Hampshire Down puros e animais mestiços Suffolk-Corriedale e Ile de FranceCorriedale. De modo semelhante, Ribeiro et al. (2000), também, não observaram diferenças entre borregos Ile de France e Hampshire Down.

Este trabalho teve como objetivos avaliar o uso de silagem de milho e do feno de aveia, na alimentação de cordeiros desmamados aos 67 dias e imediatamente confinados, bem como avaliar o desempenho de cordeiros mestiços Ile de France Corriedale, Hampshire Down - Corriedale e Suffolk - Corriedale até 130 dias de idade.

\section{Material e Métodos}

O experimento foi conduzido, entre os meses de agosto e outubro de 2000, na Fazenda Escola da Universidade Estadual de Londrina, situada no município de Londrina, Paraná. Estando localizada geograficamente a $23^{\circ} 23^{\prime} \mathrm{S}$ de latitude e $51^{\circ} 11^{\prime} \mathrm{W}$ de longitude, com altitude média de $566 \mathrm{~m}$. As temperaturas médias para os meses de agosto, setembro e outubro são de 18,$5 ; 20,0$ e $21,3^{\circ} \mathrm{C}$, e as precipitações totais médias são de 58, 105 e $179 \mathrm{~mm}$, respectivamente (CORREA; GODOY; BERNARDES, 1982). Foram utilizados um total de 30 cordeiros mestiços, sendo 10 de cada um dos seguintes grupamentos raciais: Ile de France $\mathrm{x}$ Corriedale, Hampshire Down x Corriedale e Suffolk $x$ Corriedale. Aproximadamente metade dos animais em cada grupamento era composta por machos castrados e a outra metade por fêmeas.

Os animais foram desmamados aos 67 dias de idade e distribuídos ao acaso, segundo o padrão racial e o sexo, em três lotes de 10 animais cada, sendo, então confinados em um aprisco com piso ripado e elevado do solo até atingirem 130 dias de idade. Cada lote recebeu um dos seguintes tratamentos: T1 - Volumoso composto de silagem de milho; T2 - Volumoso composto por $50 \%$ de silagem de milho $+50 \%$ de feno de aveia; e T3 -
Volumoso composto por feno de aveia. A ração concentrada suplementar foi composta com $75 \%$ de milho triturado e $25 \%$ de farelo de soja. A proporção volumoso:concentrado utilizada foi de 60:40, base da matéria seca. Os animais receberam as rações completas duas vezes ao dia, às 8:00 e às 17:00 horas.

As sobras deixadas nos cochos foram recolhidas, pesadas e amostradas diariamente antes da distribuição das rações. De maneira semelhante, amostras dos alimentos, também, foram colhidas. Dados da composição dos alimentos e da dieta são apresentados na Tabela 1.

Tabela 1. Percentagens de matéria seca (MS), proteína bruta (PB) e nutrientes digestíveis totais (NDT) dos alimentos e das dietas utilizadas, em base seca.

\begin{tabular}{lccc}
\hline & MS (\%) & PB(\%) & NDT(\%) \\
\hline Alimentos & & & \\
Silagem milho (SM) & 33,67 & 7,55 & 72,16 \\
Feno aveia (FA) & 90,61 & 9,04 & 50,68 \\
Milho grão & 88,00 & 10,57 & 90,91 \\
Farelo soja & 89,00 & 50,56 & 82,02 \\
& & & \\
Dietas & & & \\
SM + Ração concentrada & 44,74 & 12,76 & 78,77 \\
SM+FA+Ração concentrada & 59,70 & 13,20 & 72,33 \\
FA + Ração concentrada & 89,69 & 13,65 & 65,88 \\
\hline
\end{tabular}

Os alimentos foram fornecidos em quantidades a permitir sobras de $20 \%$. Conversões alimentares, em função do consumo total de alimentos pelos animais em cada período de 14 dias e dos ganhos de peso nestes períodos, foram calculadas para cada lote. Os animais tinham à disposição, em cochos apropriados, sal mineralizado e água.

Os dados para peso inicial e final e para ganho médio diário foram submetidos à análise de variância (SAS, 1994), usando-se o modelo estatístico abaixo, e as diferenças entre médias foram comparadas pelo teste " $\mathrm{t}$ ". Para a análise dos dados de consumo e conversão alimentar levou-se em consideração apenas o efeito de tratamento, pois os animais estavam alojados em baias coletivas. 
$\mathrm{Y}_{\mathrm{ijkl}}=\mu+\mathrm{T}_{\mathrm{i}}+\mathrm{G}_{\mathrm{j}}+\mathrm{S}_{\mathrm{k}}+(\mathrm{TG})_{\mathrm{ij}}+(\mathrm{TS})_{\mathrm{ik}}+(\mathrm{GS})_{\mathrm{jk}}+\mu_{\mathrm{ijkl}}$

Onde:

$\mathrm{Y}_{\mathrm{ijkl}}=$ observações para $\mathrm{o}$ animal 1 , recebendo $\mathrm{o}$ tratamento i, do grupamento racial j e do sexo k;

$\mu=$ média geral;

$\mathrm{T}_{\mathrm{i}}=$ efeito do tratamento de ordem $\mathrm{i}$, sendo $\mathrm{i}=\mathrm{T} 1$ (silagem de milho), T2 (silagem de milho + feno de aveia) e T3 (feno de aveia);

$\mathrm{G}_{\mathrm{j}=}$ efeito do grupamento racial de ordem $\mathrm{j}$, sendo $\mathrm{j}=$ IC (Ile de France x Corriedale), HC (Hampshire Down x Corriedale) e SC (Suffolk x Corriedale); $\mathrm{S}_{\mathrm{k}}=$ efeito do sexo $\mathrm{k}$, sendo $\mathrm{k}=$ macho e fêmea;
$(\mathrm{TG})_{\mathrm{ij}}=$ efeito da interação do tratamento de ordem i com o grupamento racial de ordem $\mathrm{j}$;

$(\mathrm{TS})_{\mathrm{ik}}=$ efeito da interação do tratamento de ordem i com o sexo do cordeiro de ordem $\mathrm{k}$;

$(G S)_{j k}=$ efeito da interação do grupamento racial de ordem j com o sexo do cordeiro de ordem k;

$\mu_{\mathrm{ijkl}}=$ erro aleatório associado a cada observação.

\section{Resultados e Discussão}

Resultados para consumo de alimentos em percentagem do peso vivo ou por unidade de tamanho metabólico e para a conversão alimentar, segundo os tratamentos, são apresentados na Tabela 2.

Tabela 2. Médias e erros padrões para consumo de alimentos por unidade de tamanho metabólico (UTM) e em percentagem do peso vivo (PV) e conversão alimentar (consumo em kg/ganho médio diário em $\mathrm{kg}$ ), segundo os tratamentos.

\begin{tabular}{lccc}
\hline Tratamento & Consumo-UTM, g & Consumo-PV, \% & Conversão \\
\hline Silagem de milho (SM) & $97,36 \pm 3,08 \mathrm{~b}$ & $2,84 \pm 0,09 \mathrm{~b}$ & $4,88 \pm 1,14$ \\
SM + FA & $108,46 \pm 3,08 \mathrm{a}$ & $3,16 \pm 0,09 \mathrm{a}$ & $5,06 \pm 1,17$ \\
Feno de aveia (FA) & $98,11 \pm 3,08 \mathrm{~b}$ & $2,88 \pm 0,09 \mathrm{~b}$ & $4,31 \pm 0,94$ \\
Pr $>$ F & 0,0327 & 0,0353 & 0,8561 \\
\hline
\end{tabular}

$\mathrm{a}, \mathrm{b}$ - Médias na coluna seguidas por letras diferentes diferem entre si $(\mathrm{P}<0,05)$.

Pode ser observado que nas duas formas de expressar consumo, os animais que receberam como fonte volumosa a combinação de silagem de milho com feno de aveia, apresentaram consumo de matéria seca superior aos observados com os dos animais que receberam exclusivamente silagem de milho ou feno de aveia como fontes de volumosos. Esta combinação de volumosos provavelmente tornou a ração mais apetecível aos animais, porém, em termos de composições químicas quantificadas nas rações não foram averiguadas justificativas claras para que isto tenha ocorrido, já que os valores para matéria seca, proteína bruta e NDT, foram intermediários aos outros dois tratamentos (Tabela 1). Os animais que receberam silagem de milho ou feno de aveia apresentaram consumos de matéria seca muito próximos e não diferiram entre si. De maneira concordante, consumos similares para cabras recebendo silagem de milho e feno de aveia foram observados por Ribeiro (2000).

Estes resultados, de certa forma, concordam com os encontrados por Codagnone et al. (1988) para vacas leiteiras, que observaram maior consumo de matéria seca por unidade de tamanho metabólico, em animais que receberam uma combinação destes dois volumosos na proporção de $25 \%$ de silagem de milho e $75 \%$ de feno de aveia. Os menores consumos de matéria seca foram observados para os animais que receberam $100 \%$ de volumoso em silagem de 
milho ou feno de aveia, não havendo diferença entre eles (CODAGNONE et al., 1988). Outras combinações apresentaram resultados intermediários. Em relação ao menor consumo de matéria seca de silagem, os autores supracitados afirmam que a mesma apresenta fatores depressores do consumo, como o pH e a umidade. Uma possível explicação para o menor consumo de matéria seca observado com os animais que receberam feno de aveia (lote T3) é o seu menor conteúdo energético que pode ser observado na Tabela 1. Rodrigues, Rodrigues e Branco (2001) observaram que cabras em lactação apresentaram um maior consumo de matéria seca para dietas com 1,7 e 1,4 Mcal/kg em comparação àquelas alimentadas com dietas contendo $1,0 \mathrm{Mcal} / \mathrm{kg}$.

A conversão alimentar foi semelhante entre os tratamentos, sendo que as médias variaram de 4,31 a 5,06. Estas médias foram menores do que 6,18 de conversão média observada por Pilar et al. (1994) com ovinos de diversos grupamentos genéticos, alimentados com silagem de milho como fonte volumosa. De maneira similar, os resultados observados no presente trabalho são melhores do que aqueles observados por Ribeiro et al. (2002), que trabalharam com ovelhas adultas Hampshire Down recebendo diferentes silagens como fonte de volumosos, e divulgaram conversões alimentares que variaram de 6,14 a 7,96. Porém, nestes dois últimos experimentos os autores trabalharam com animais mais velhos do que no presente estudo. Por outro lado, Yamamoto (2003), trabalhando com animais com idade semelhante à do presente estudo, porém com dietas com menor proporção de volumosos (32\%), observaram conversões alimentares que variaram de 3,51 a 3,96. Orskov (1982, apud Siqueira 1996), comenta que a conversão depende entre outras coisas, do nível de consumo , da composição do ganho, do sexo e do potencial genético para ganho de peso. Para taxas de ganho de peso de 100 g/dia as conversões citadas pelo autor são de 5,9 e 5,5 para machos e fêmeas, respectivamente, e para ganhos de $200 \mathrm{~g} / \mathrm{dia}$, a conversão, média para os dois sexos, é de 3,9.

Em relação as médias para consumo, os valores expressos em percentagem do peso vivo foram próximos aos citados por Pilar et al. (1994) e Ribeiro et al. (2002). Porém, quando os valores foram expressos em gramas por unidade de tamanho metabólico, os consumos observados no presente trabalho foram maiores do que aqueles citados pelos referidos autores. Greenhalgh (1982, apud Siqueira 1996) menciona consumos de matéria seca em gramas por unidade de tamanho metabólico de 57,0 e 90,5 para dietas fibrosas (forragens não tratadas) e finas (concentrados, forragens peletizadas ou raízes), respectivamente.

As médias para peso inicial (67 dias de idade), peso final (130 dias de idade) e ganho médio diário neste período são apresentadas na Tabela 3, sendo que não houve interação $(\mathrm{P}>0,10)$ entre as fontes de variação estudadas (tratamentos, grupos raciais e sexo dos cordeiros). Pode ser observado que não houve diferença estatística entre os tratamentos, ou seja, silagem de milho, silagem de milho + feno de aveia e feno de aveia, para o peso inicial e final. Para o ganho médio diário, apesar da aparente vantagem dos animais alimentados com feno de aveia sobre os alimentados com silagem de milho, esta diferença $(17,9 \%)$ não foi significativa. Estes resultados concordam com os observados para produção de leite por Codagnone et al. (1988), trabalhando com vacas leiteiras, e Ribeiro (2000) trabalhando com cabras leiteiras, que não observaram diferenças na produção de animais recebendo silagem de milho ou feno de aveia como fonte volumosa. 
Tabela 3. Médias e erros padrões para pesos e ganhos médios diários (GMD) do desmame aos 130 dias de idade em cordeiros, segundo os tratamentos, grupos raciais e sexos.

\begin{tabular}{|c|c|c|c|}
\hline Efeitos & $\begin{array}{c}\text { Peso Inicial } \\
(\mathrm{kg})\end{array}$ & $\begin{array}{c}\text { Peso Final } \\
(\mathrm{kg})\end{array}$ & $\begin{array}{c}\text { GMD } \\
(\mathrm{kg})\end{array}$ \\
\hline \multicolumn{4}{|l|}{ Tratamento } \\
\hline Silagem de milho (SM) & $11,51 \pm 1,45$ & $17,53 \pm 1,67$ & $0,095 \pm 0,012$ \\
\hline $\mathrm{SM}+\mathrm{FA}$ & $12,46 \pm 1,49$ & $19,34 \pm 1,71$ & $0,109 \pm 0,012$ \\
\hline Feno de aveia (FA) & $12,33 \pm 1,20$ & $19,35 \pm 1,38$ & $0,112 \pm 0,010$ \\
\hline $\operatorname{Pr}>F$ & 0,8888 & 0,6830 & 0,5986 \\
\hline \multicolumn{4}{|l|}{ Grupo racial } \\
\hline Hampshire Down & $13,07 \pm 0,90$ & $18,98 \pm 1,03$ & $0,094 \pm 0,007 \mathrm{~b}$ \\
\hline Ile de France & $11,38 \pm 1,59$ & $17,30 \pm 1,83$ & $0,094 \pm 0,013 \mathrm{~b}$ \\
\hline Suffolk & $11,85 \pm 1,59$ & $19,94 \pm 1,38$ & $0,128 \pm 0,013 \mathrm{a}$ \\
\hline $\operatorname{Pr}>\mathrm{F}$ & 0,5798 & 0,6078 & 0,1093 \\
\hline \multicolumn{4}{|l|}{ Sexo } \\
\hline Fêmea & $12,21 \pm 0,89$ & $17,93 \pm 1,03$ & $0,091 \pm 0,007 \mathrm{~B}$ \\
\hline Macho & $11,99 \pm 1,36$ & $19,55 \pm 1,57$ & $0,120 \pm 0,011 \mathrm{~A}$ \\
\hline $\operatorname{Pr}>F$ & 0,8953 & 0,4197 & 0,0571 \\
\hline
\end{tabular}

a, $\mathrm{b}$ - Médias na coluna, para o mesmo efeito, seguido por letras diferentes diferem entre si $(\mathrm{P}<0,11)$.

A, B - Médias na coluna, para o mesmo efeito, seguido por letras diferentes diferem entre si $(\mathrm{P}<0,06)$.

Em relação aos grupamentos raciais, apesar de não ter havido diferença significativa para peso inicial e final, cordeiros mestiços Suffolk ganharam mais peso $(36,2 \%)$ do que cordeiros mestiços Hampshire Down e Ile de France. Sendo que cordeiros destas últimas duas raças apresentaram exatamente o mesmo ganho de peso (Tabela 3). Estes resultados concordam parcialmente com os apresentados por Kempster et al. (1987), que apesar de observarem maiores pesos ao abate em borregos mestiços Suffolk, não observaram diferenças no ganho de peso entre estes cordeiros e os mestiços Hampshire Down e mestiços Ile de France. Os resultados observados por Pilar et al. (1994) e Ribeiro et al. (2000) corroboram com os achados de Kempster et al. (1987).

Com relação ao sexo dos cordeiros, pode ser observado que os machos tiveram um ganho de peso $31,9 \%$ maior do que as fêmeas, porém não houve diferença estatística para os pesos inicial e final (Tabela 3). Segundo Figueiró e Benavides (1990) cordeiros geralmente ganham mais peso do que cordeiras, e quanto mais velhos os animais, maior a diferença. Esta diferença tem a ver com a maior produção de hormônios sexuais (andrógenos) pelos testículos após a puberdade dos animais, o que ocorre normalmente entre 6 a 9 meses de idade, fase influenciada pelo genótipo do animal, fotoperíodo, temperatura, nutrição e condição corporal (MUKASA-MUGERWA; EZAZ, 1992; CASTRILLEJO et al., 1995; PRICE; BORGWARDT; DALLY, 1995). Carvalho et al. (1997) não observaram diferenças no peso e no ganho de peso de cordeiros e cordeiras até os 100 dias de idade. Por outro lado, Roda et al. (1990) observaram que cordeiros foram mais pesados do que cordeiras ao desmame, aos 84 dias de idade.

\section{Conclusões}

Não houve diferença no consumo de matéria seca e no ganho de peso para ovinos alimentados com silagem de milho ou feno de aveia como fontes volumosas.

Cordeiros mestiços Suffolk ganharam mais peso em confinamento até os 130 dias de idade do que cordeiros mestiços Hampshire Down ou Ile de France.

Machos ganharam mais peso do que fêmeas até os 130 dias de idade.

\section{Referências}

AMARANTE, A. F. T. Profilaxia da verminose ovina, descontaminação de pastagens. In: SILVA SOBRINHO, A.G. (Ed.). Produção de ovinos. Jaboticabal: FUNEP, 1990. p.201-210.

CARTER, A. H.; KIRTON, A. H. Lamb production performance of 14 sire breeds mated to New Zealand Romney ewes. Livestock Production Science, Amsterdam, v.2, p.157-166, 1975.

CARVALHO, S.; PIRES, C.C.; SACCOL, A.G.; MÜLLER, M. Confinamento de cordeiros machos inteiros, castrados e fêmeas abatidos aos 100 dias de idade. In: REUNIÃO ANUAL DA SOCIEDADE BRASILEIRA DE ZOOTECNIA, 34., 1997, Juiz de Fora. Anais... Viçosa: Soc. Bras. Zootec., 1997. CD-Rom.

CASTRILLEJO, A.; MORANA, A.; BIELLI, A.; GASTEL, T.; MOLINA, J.R.; FORSBERG, M.; RODRIGUEZMARTINEZ, H. Onset of spermatogenesis in Corriedale ram lambs under extensive rearing conditions in Uruguay. Acta Veterinaria Scandinavica, Copenhagen, v.36, n.2, p.161-173, 1995. 
CODAGNONE, H.C.V.; CARDOSO, R.M.; CASTRO, A.C.G.; SILVA, M.A. Silagem de milho e feno de aveia (Avena bizantina L.) na alimentação de vacas em lactação. Revista da Sociedade Brasileira de Zootecnia, Viçosa, v.17, n.6, p.487-497, 1988.

CORRÊA, A.R.; GODOY, H.; BERNARDES, L.R.M. Características climáticas de Londrina. 2.ed. Londrina: IAPAR, 1982. $16 \mathrm{p}$.

FIGUEIRÓ, P.R.P.; BENAVIDES, M.V. Produção de carne ovina. In: Caprinocultura e ovinocultura. Piracicaba: Soc. Bras. Zootec.; FEALQ, 1990. p.15-31.

KEMPSTER, A.J.; CROSTON, D.; GUY, D.R.; JONES, D.W. Growth and carcass characteristics of crossbred lambs by ten sire breeds, compared at the same estimated carcass subcutaneous fat proportion. Animal Production, Bletchley, v.44, p.83-98, 1987.

LUPATINI, G.C.; NUNES, S.P. Milho para produção de silagem de qualidade. In: RESTLE, J. (Ed.). Confinamento, pastagens e suplementação para produção de bovinos de corte. Santa Maria: UFSM, 1999. p.104-124.

MACEDO, F.A.F.; SIQUEIRA, E.R.; MARTINS, E.N. Desempenho de cordeiros Corriedale puros e mestiços, terminados em pastagem e confinamento. In: REUNIÃO ANUAL DA SOCIEDADE BRASILEIRA DE ZOOTECNIA, 35., 1998, Botucatu. Anais... Viçosa: Soc. Bras. Zootec., 1998a. p.636-638.

MACEDO, F.A.F; SIQUEIRA, E.R.; MARTINS, E.N. Análise econômica da produção de carne de cordeiros sob dois sistemas de terminação: Pastagem e confinamento. In: REUNIÃO ANUAL DA SOCIEDADE BRASILEIRA DE ZOOTECNIA, 35., 1998, Botucatu. Anais... Viçosa: Soc. Bras. Zootec., 1998b. p.645-647.

MILLIGAN, K. Princípios y prácticas para el manejo intensivo de lanares. Montevideo: Hemisfério Sur, 1985. $84 \mathrm{p}$.

MUKASA-MUGERVWA, E.; EZAZ, Z. Relationship of testicular growth and size to age, body weight and onset of puberty in Menz ram lambs. Theriogenology, Stoneham, v.38, n.5, p.979-988, 1992.

PILAR, R.C.; PIRES, C.C.; RESTLÉ, J.; SILVEIRA, S.S.; GONÇALVES, J.M.; FERNANDES, F. Desempenho em confinamento e componentes do peso vivo de diferentes genótipos de ovinos abatidos aos doze meses de idade. Ciência Rural, Santa Maria, v.24, n.3, p.607-612, 1994.

PRICE, E. O.; BORGWARDT, R.; DALLY, M. R. Heterossexual experience differentially affects the expression of sexual behavior in 6- and 8-month-old ram lambs. Applied Animal Behaviour Science, Amsterdam, v.46, n.3/4, p.193-199, 1995.
RIBEIRO, L. R. Consumo, produção e composição do leite e parâmetros sanguíneos de cabras leiteiras alimentadas com dietas, contendo diferentes fontes de volumosos. Maringá, PR: Universidade Estadual de Maringá - UEM, 2000. 29 p. Dissertação (Mestrado em Zootecnia) Universidade Estadual de Maringá, 2000.

RIBEIRO, E. L. A.; ROCHA, M. A.; MIZUBUTI, I. Y.; MORI, R. M. Ganho de peso e componentes do peso vivo em borregos Ile de France inteiros ou castrados e Hampshire Down castrados abatidos aos doze meses de idade. Ciência Rural, Santa Maria, v.30, n.2, p.333-336, 2000.

RIBEIRO, E. L.A.; ROCHA, M.A.; MIZUBUTI, I.Y.; SILVA, L.D.F. Silagens de girassol (Helianthus annus L.), milho (Zea mays L.) e sorgo (Sorghum bicolor L.) Moench) para ovelhas em confinamento. Ciência Rural, Santa Maria, v.32, n.2, p.299-302, 2002.

RODA, D.S.; SANTOS, L.E.; DUPAS, W.; CUNHA, E. A.; FEITOSA, A.S.L. Avaliação do peso ao nascer e ao desmame e mortalidade pré-desmame em cordeiros Santa Inês, Suffolk e cruzados Suffolk. Boletim de Indústria Animal, Nova Odessa, v.47, n.2, p.153-157, 1990.

RODRIGUES, C. A. P. F.; RODRIGUES, M. T.; BRANCO, R. H. Influencia do nível energético da dieta sobre o consumo de cabras Alpinas durante o pré parto. In: REUNIÃO ANUAL DA SOCIEDADE BRASILEIRA DE ZOOTECNIA, 38., 2001, Piracicaba. Anais... Viçosa: Soc. Bras. Zootec., 2001. CD-Rom.

SÁ, J.P.G. Utilização da aveia na alimentação animal. Londrina: IAPAR, 1995. 19 p.

SAS INSTITUTE. SAS/STAT: User's Guide. Cary, 1994. v. 2,846 p.

SIQUEIRA, E. R. Recria e terminação de cordeiros em confinamento. In: SILVA SOBRINO, A.G. (Ed.). Nutrição de ovinos. Jaboticabal: FUNEP, 1996. p.175-2121.

SIQUEIRA, E.R.; AMARANTE, A.F.T.; FERNANDES, S. Estudo comparativo da recria de cordeiros em confinamento e pastagem. Veterinária e Zootecnia, São Paulo, v.5, p.17-28, 1993.

YAMAMOTO, S.M. Desempenho, digestibilidade e características quantitativas e qualitativas de carcaças de cordeiros Santa Inês puros e mestiços, terminados em confinamento com dietas contendo diferentes fontes de óleo vegetal. Maringá, PR: Universidade Estadual de Maringá - UEM, 2003. 74 p. Dissertação (Mestrado em Zootecnia) - Universidade Estadual de Maringá, 2003. 
